CSIC, Madrid.

COMELLES, M. - 1981-Contribució al coneixement de les carofícies d’España. Collect. Bot. (Barcelona) 12: 97-103.

CORILLION, R. -1961- Les végétations précoces de charophycées d'Espagne méridionale et du Maroc Occidental. Rev. Gen. Bot. 68: 317-331.

DAVIES-COLLEY, R.J. - 1988- Measuring water with a black-disk. Limnol. Oceanog. 33: 616623.

FELDMAN, G. -1945- Deux nouvelles espèces de Chara de l'Afrique du Nord. Bull. Soc. Hist. Nat. Afrique Nord 36: 168-173.

GARCÍA MURILLO, P., M. BERNUÉS y C. MONTES. -1993-Los macrófitos acuáticos del Parque Nacional de Doñana (SW España).
Aspectos florísticos. Actas VI Congreso español de Limnología: 261-267.

WOOD, R. D. \& K. IMAHORI -1965-Monograph of the Characeae. Verlag Von J. Cramer, Weinheim.

Aceptado para su publicación en Abril de 1997

Dirección de los autores. J. L. Espinar y P. García Murillo: Departamento de Biología Vegetal y Ecología, Facultad de Farmacia, Universidad de Sevilla. Apartado 874. 41071 Sevilla. S. Cirujano: Real Jardín Botánico, CSIC. Plaza de Murillo, 2. 28014 Madrid.

\title{
59. CONTRIBUCIONES AL MACROFITOBENTOS DEL MUNICIPIO DE CAMAÇARI (BAHÍA, BRASIL)
}

María ALTAMIRANO y José Marcos de Castro NUNES

Contributions to the macrophytobenthos from Camaçari (Bahia, Brasil)

Palabras clave. Algas, Bahía, Brasil, catálogo, macrofitobentos.

Key words. Bahia, Brasil, check-list, macrophytobenthos, seaweeds.

El Estado de Bahía es el que posee el litoral más extenso de todo Brasil. El macrofitobentos de esta costa está poco conocido. Los primeros trabajos (Martius et al., 1883; Martens, 1870; Dickie, 1874; Moebius, 1889,1890; Howe, 1928; Taylor, 1931) son lejanos en el tiempo, y sólo recientemente se ha llevado a cabo el primer catálogo del macrofitobentos del litoral bahiano, que incluía exclusivamente a las clorofíceas (Vasques Martins et al., 1991). En la actualidad, se siguen elaborando inventarios de distintos puntos del litoral bahiano aún sin estudiar, como es el caso de la Playa de Itaçimirin, Municipio de Camaçari $\left(12^{\circ} 42^{\prime} \mathrm{S}\right.$ $38^{\circ} 20^{\prime} \mathrm{W}$ ) (fig. 1). Esta zona está situada en el litoral Norte y presenta tanto sustratos rocosos como arenosos. El objetivo de este trabajo es la elaboración del catálogo de las macroalgas marinas de la Playa de Itaçimirin. 
212

Acta Botanica Malacitana 22. 1997

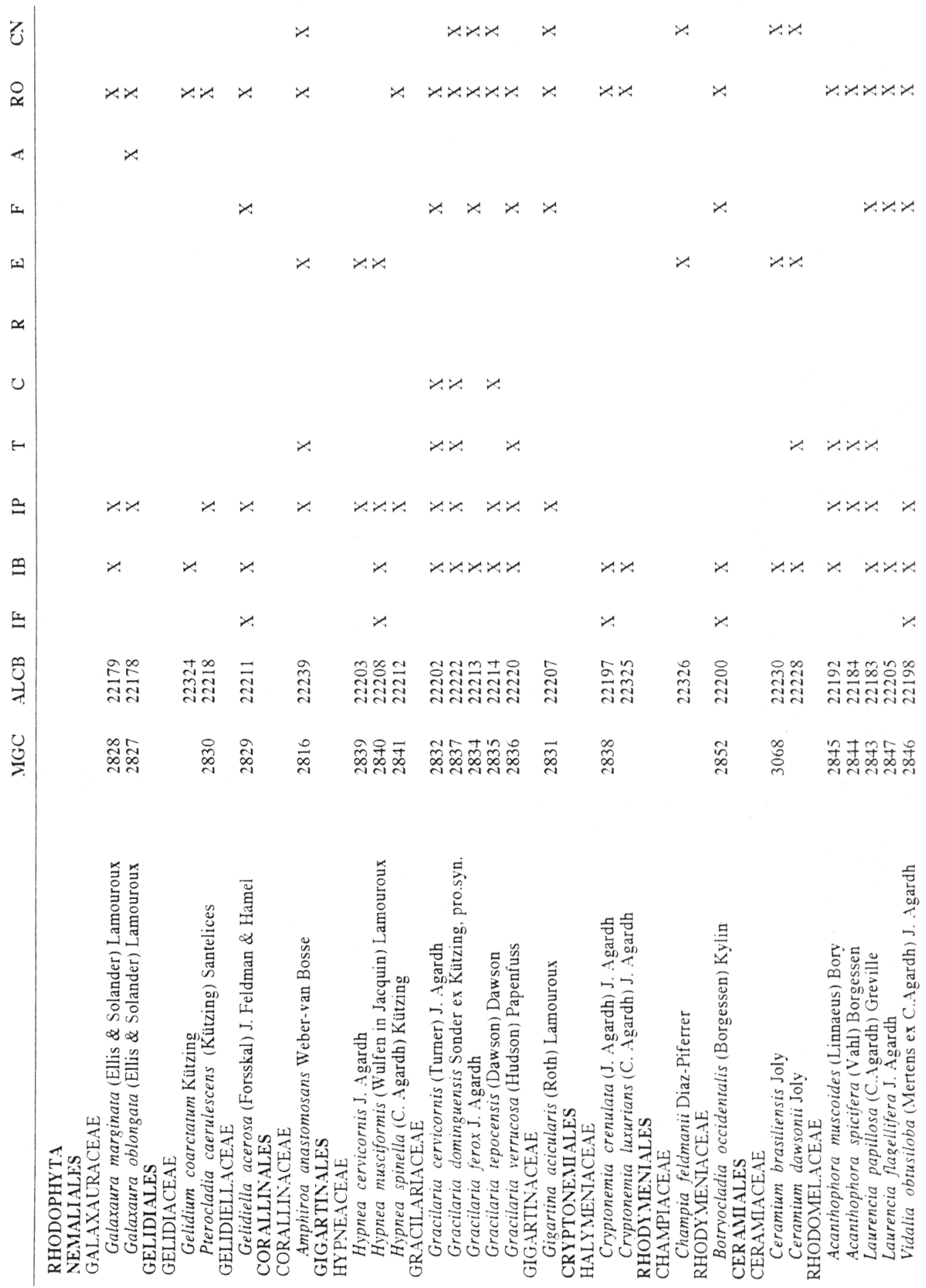




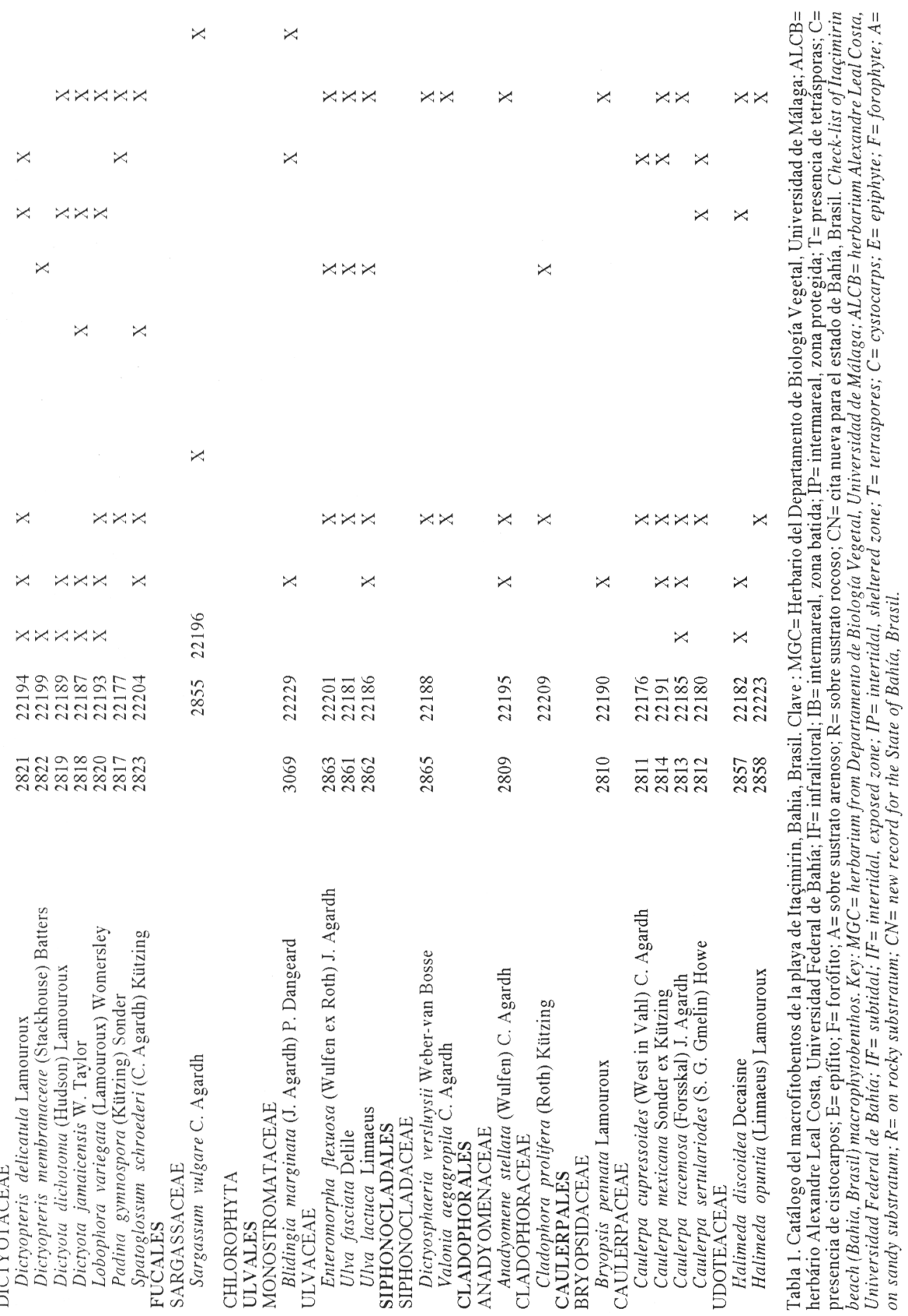




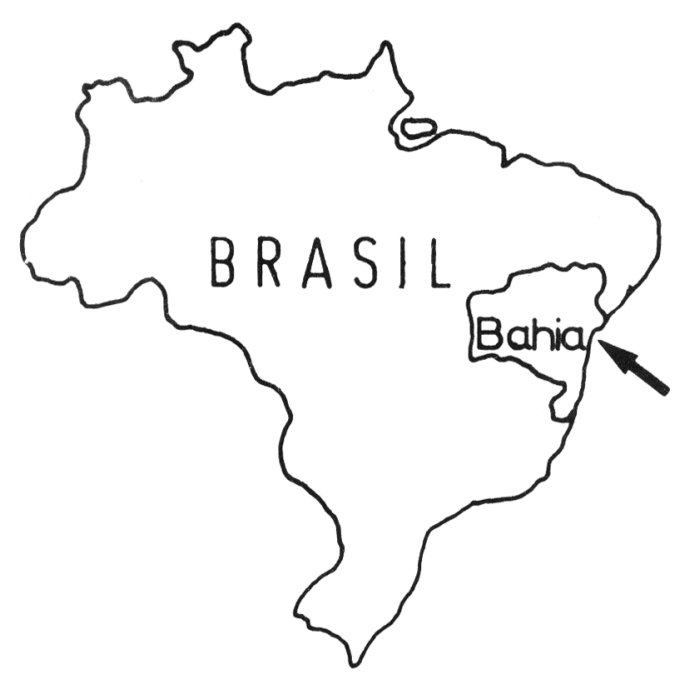

Figura 1.- Situación geográfica de la playa de Itaçimirin (Bahía, Brasil).

Las muestras se recogieron en Agosto de 1995, aprovechando las horas de bajamar. Se consideraron tres subdivisiones en cuanto al hábitat de las algas: dos en la zona intermareal (costa protegida y costa expuesta al oleaje) y la zona infralitoral. Las muestras se preser̃varon en formol al $4 \%$ en agua de mar. Para la identificación se siguió a Taylor (1960), Joly (1965, 1967), Cordeiro-Marino (1978) y Vasques et al. (1991). Tras su identificación, se pasó a su prensado, depositándose un ejemplar en los herbarios ALCB (Herbário Alexandre Leal Costa, Universidad Federal de Bahía, Salvador de Bahía) y MGC (Departamento de Biología Vegetal, Universidad de Málaga). Los taxones aparecidos se han ordenado según los criterios nomenclaturales de Wynne (1986).

Los resultados se recogen en la tabla 1 , donde se anota el número de referencia en el herbario MGC y en el herbario ALCB, la zona en la que fueron encontrados los ejemplares (IF = infralitoral IB = intermareal, zona batida; IP = intermareal, zona protegida), el estado reproductivo $(\mathrm{T}=$ tetrásporas; $\mathrm{C}=$ cistocarpos; $\mathrm{R}=$ esporangios), si era epífito (E) o forófito $(\mathrm{F})$, el sustrato sobre el que se encontró $(\mathrm{A}=$ arena; $\mathrm{RO}=$ roca) y si es cita nueva para todo el litoral del Estado de Bahía (CN).

En total se identificaron 49 especies $(25$ Rodófitas, 8 Feófitas y 15 Clorófitas), 9 de las cuales son citas nuevas para el Estado de Bahía (Amphiroa anastomosans, Gracilaria dominguensis, Gracilaria ferox, Gracilaria tepocensis, Gigartina acicularis, Champia feldmanii, Ceramium brasiliense, Ceramium dawsonii y Blidingia marginata).

AGRADECIMIENTOS. M. A. disfrutó una beca Intercampus del Instituto de Cooperación Iberoamericana en la Universidad Federal de Bahía. Los autores agradecen sinceramente al Dr. Antonio Flores-Moya su desinteresada y valiosa ayuda en la revisión definitiva del manuscrito, así como a Belem, Silvana y Cid por su caluroso apoyo en tierras tan lejanas.

\section{BIBLIOGRAFÍA}

CORDEIRO-MARINO, M. -1978- Rodofíceas bentônicas marinhas do Estado de Santa Catarina. Rickia 7 : 243 pp.

DICKIE, G. -1874-Enumeration of algae collected from Bahia by H. N. Mosely, M. A. naturalist to H. M. S. «Chalenger». Journal of the Linnean Society of London, Botany 14: 377.

HOWE, M.A. -1928- Notes on some marine algae from Brazil and Barbados. Journal of the Washington Academy of Science 18 (7): 186194.

JOLY, A.B. -1965- Flora marinha do Litoral Norte do Estado de Sao Paulo e Regioes circunvizinhas. Separata do Boletim n.294. Fac. Fil., Ciênc. e Letras da USP Botânica 21 : 1 393.

JOLY, A.B. -1967-Géneros de algas marinhas da Costa Atlântica latinoamericana. Editora da Universidade de Sao Paulo.

MARTENS, G. von. -1870- Conspectus algarum Brasiliae hactenus detectarum. Videnskabelige 
Meddelelser fra Dansk Naturhistorik Forening i Kjobenhavn 2: 297-314.

MOEBIUS, M. -1889- Bearbeitung der von $\mathrm{H}$. Schenck in Brasilien gesammelten Algen. Hedwigia 28(5): 309-347.

MOEBIUS, M. -1890- Algae brasiliensis a Dr. Glaziou collectae. Notarisia 5(20): 1065-1090. TAYLOR, W.R. -1960- Marine algae of Eastern tropical and subtropical coasts of the Americas. The University of Michigan Press.

VASQUES MARTINS, D., M. CORDEIROMARINO, N. BARBOSA BOCCANERA y J.M. CASTRO NUNES - 1991-Clorofíceas marinhas bentônicas do Munícipio de Salvador, Bahia, Brasil. Hoehnea 18(2): 115-133.
WYNNE, M.J. - 1986- A check-list of benthic marine algae of the tropical and subtropical western Atlantic. Canadian Journal of Botany 64: 22392281 .

Aceptado para su publicación en Mayo de 1997

Dirección de los autores. M. ALTAMIRANO: Departamento de Biología Vegetal. Facultad de Ciencias. Universidad de Málaga. Campus de Teatinos s/n. E-29071 Málaga. España. J.M. de CASTRO NUNES: Departamento de Botânica, Instituto de Biologia da Universidade Federal da Bahia. Campus Universitario de Ondina. CEP 40.170-210, Salvador, Bahia, Brasil.

\title{
60. REFERENCIAS COROLÓGICAS DE PLANTAS VASCULARES PARA EL SE. IBÉRICO
}

\author{
Arturo VALDÉS FRANZI y Rafael MOLINA CANTOS
}

Geographical distribution references of vascular plants for Southeastern Spain.

Palabras clave. Plantas vasculares, corología, SE. España.

Key words. Vascular plants, geographical distribution, Southeastern Spain.

Se ofrece una relación de plantas vasculares recolectadas en su mayoría en el $\mathrm{N}$. de Albacete, España. Las especies señaladas con un asterisco constituyen novedades de ámbito provincial. El resto son segundas citas para la provincia de Albacete de plantas poco frecuentes que amplían el área conocida para dicha especie en más de $100 \mathrm{~km}$ y, en algún caso, confirman citas de autores antiguos.

Los taxones aparecen en orden alfabético. La nomenclatura y autorías de los mismos siguen a Flora Iberica (Castroviejo et al., 19861993) o, si no han sido publicados aún en dicha obra, a Flora Europaea (Tutin et al., 19641980).

Se conservan tusigos de todas las citas en el herbario ALBA de la Escuela Universitaria de Magisterio, Plaza de la Universidad, Campus Universitario. Albacete. España.

*Alnus glutinosa (L.) Gaertner CUENCA: Casas de Bénitez, La Losa, presa, 